\title{
The Composition of Bovine Peritubular Dentin: Matching TOF-SIMS, Scanning Electron Microscopy and Biochemical Component Distributions
}

\author{
New Light on Peritubular Dentin Function
}

\author{
Bat Ami Gotliv ${ }^{a} \quad$ Arthur Veis $^{b}$ \\ ${ }^{a}$ Department of Chemical Physics, Weizmann Institute of Science, Rehovot, Israel; ${ }^{b}$ Department of Cell and \\ Molecular Biology, Feinberg School of Medicine, Northwestern University, Chicago, Ill., USA
}

\section{Key Words}

Peritubular dentin - Time of flight mass spectrometry • Collagen $\cdot$ Matrix proteins $\cdot$ Amino acids $\cdot$ Phospholipids

\begin{abstract}
Peritubular dentin (PTD) is a hypermineralized phase within the dentinal tubules in some vertebrate teeth as an interface between the intertubular dentin (ITD) and the cell processes. Our aim has been to understand the composition, structure and role of PTD as a mineralized tissue. We have utilized the technique of time of flight secondary ion mass spectrometry (TOF-SIMS) to map the distribution of positive and negative inorganic ions as well as organic components in the fully mineralized, intact PTD structure in bovine tooth cross-sections, and correlated these with scanning electron microscopy (SEM) in standard and backscatter modes. In recent work, we developed a procedure to freeze fracture the teeth
\end{abstract}

\begin{tabular}{ll}
\hline Abbreviations used in this paper \\
\hline ITD & intertubular dentin \\
PTD & peritubular dentin \\
SEM & scanning electron microscopy \\
TOF-SIMS & time of flight secondary ion mass spectrometry \\
\hline
\end{tabular}

\section{KARGER}

Fax +41613061234 E-Mail karger@karger.ch www.karger.com
(C) 2008 S. Karger AG, Basel www.karger.com/cto and separate PTD from the less dense ITD by the use of aqueous sodium phosphotungstate step density gradients, after degrading the ITD collagen with $\mathrm{NaOCl}$. Here, PTD-containing fragments were characterized by SEM and TOF-SIMS surface structure analysis. The TOF-SIMS data show that the isolated PTD does not contain collagen, but its surface is rich in glutamic acid-containing protein(s). The TOF-SIMS spectra also indicated that the intact PTD fragments contain phospholipids, and chemical analyses showed phosphatidylserine, phosphatidylinositol and phosphatidylcholine as the principal lipid components. In SEM sections, untreated PTD shows as a smooth collar around the tubule, but after digestion with ethylenediamine to remove all organic components, the porous nature of the mineral phase of small, thin platy apatite crystals becomes evident. Thus, the organic matrix of PTD appears to be a proteolipid-phospholipid complex.

Copyright $\odot 2008$ S. Karger AG, Basel

\section{Introduction}

A major aim of studies of mineralized tissues has been to determine the components of the system and understand how they relate to each other to create the tissue architecture and regulate the formation of the mineral phase. Biochemical and molecular biology studies have

Tel. +1 312503 1355, Fax +1 312503 2544, E-Mail aveis@northwestern.edu 
provided information on the major molecules and their characteristics, while physical techniques, such as X-ray crystallography, have described the mineral crystals and how they are packed and organized. However, since the biochemical approaches require the disruption and dissolution of the tissue in order to collect the matrix components, their correlation with each other in position and function has generally had to be deduced by indirect means. For example, in mineralized tissues, component localization by means of immunocytochemistry has uncertainties, since the mineral phase must be removed to expose the epitopes of the relevant matrix macromolecules, and the procedures used for this may introduce artifacts. Thus, proteins occluded in the mineral phase, but not accessible during tissue fixation could be missed as important modulators of the physical properties of the mineral phase. Techniques of surface analysis in metallurgy and surface chemistry have been developed which are applicable to the in situ analysis of mineralized tissues without prior degradative treatments. In the present study, one of these methods, time of flight secondary ion mass spectrometry (TOF-SIMS), has been applied in concert with biochemical analyses and other physical methods to examine the composition of the organic components of bovine peritubular dentin (PTD) compared with the composition of intertubular dentin (ITD).

\section{Materials and Methods}

\section{TOF-SIMS Features}

In TOF-SIMS, a focused high-energy pulsed beam (nanosecond pulses) of metal ions, such as gallium $\left(\mathrm{Ga}^{+}\right)$or gold $\left(\mathrm{Au}^{+}\right)$, is rastered across the surface of a sample. These ions penetrate the surface and transfer their energy to the molecules in the surface, resulting in the formation of charged ions originating within and depending upon the composition of the material. These secondary ions are ejected from the surface and drawn into the mass spectrometer, where their mass is analyzed by their time of flight from the surface to the detector. The ejected secondary ions may be simple, monoatomic ions, such as $\mathrm{Ca}^{+}$or $\mathrm{Mg}^{+}$, or more complex structures from the breakdown of organic molecules. Each pixel on the surface releases a spectrum describing the masses of all ions originating within that area, and the composition of the released ions depends on the depth of penetration of the primary beam. In order to map the surface, the density of the beam is kept low, in the order of $10^{12}$ ions $/ \mathrm{cm}^{2}$, much lower than the approximately $10^{15}$ atoms $/ \mathrm{cm}^{2}$ residing in a monolayer. Thus, differential sampling of the spectrum from adjacent pixels by the pulsed beam is possible. The sampling depth of static SIMS is only a few atom diameters, 10-20 A. Thus, a protein molecule with a dimension of greater than $40 \AA$ would be only partially interacting to give rise to charged fragments, ranging from single amino acid fragments to larger polypeptides. In commercial instruments avail- able, the upper limit to organic fragment size is about 2,000 Da. The mass resolution of TOF-SIMS is superb, at $10,000(\mathrm{~m} / \Delta \mathrm{m})$, so that ion fragments differing by only a few millimass units can be reliably distinguished from each other. In practice, the spectrum will contain many mass fragments of different intensity. The investigator must select the most pertinent characteristic fragment masses to follow for determining the distribution of sample surface constituents.

\section{Data Acquisition}

A PHI THRIFT III (Physical Electronics, Chanhassen, Minn., USA) TOF-SIMS apparatus was used with a liquid Ga primary ion source, producing a $25-\mathrm{keV}$ ion beam. After the sample was mounted, the surface was cleaned of absorbed substances, and smoothed by sputtering with the $\mathrm{Ga}^{+}$beam for $1 \mathrm{~min}$. Spectra were then acquired over a $30 \times 30 \mu \mathrm{m}$ surface area $(256 \times 256$ pixels) in both positive and negative ion modes. The masses were calibrated with $\mathrm{CH}_{3}{ }^{+}, \mathrm{C}_{2} \mathrm{H}^{+}, \mathrm{C}_{3} \mathrm{H}_{5}{ }^{+}, \mathrm{CH}^{-}, \mathrm{OH}^{-}$and $\mathrm{C}_{2} \mathrm{H}^{-}$ions. Amino acid fragment ions were identified from the SIMS spectra of poly- $\alpha$ amino acids absorbed on silica wafers [Mantus et al., 1993; Samuel et al., 2001; Dambach et al., 2004], phospholipid fragment ion identification was based on the data of Ostrowski et al. [2005]. Since the polypeptides and phospholipids yield many fragments, we selected those fragments shown in earlier studies to have the highest signal intensities, at masses minimally overlapping with ion fragments originating from other components. For example, the $\mathrm{C}_{2} \mathrm{H}_{6} \mathrm{~N}^{+}$fragment is the most intense peak $(\mathrm{M}=$ 44.05) from both Ala and Ser. A less intense, but not overlapping peak at $M=71$ from Ser was used to distinguish between these amino acids.

\section{Bovine Tooth and PTD Preparation}

Unerupted and erupted bovine molar teeth were collected, placed on ice and processed as described [Gotliv and Veis, 2007] as rapidly as possible. The intact teeth were divided into coronal and root portions using an Isomet 1000 diamond saw. The coronal sections were embedded in epon and cut into thin sections to expose surfaces for hand polishing with 4,000-grit paper followed by $0.3-\mu \mathrm{m}$ aluminum paste [Gotliv et al., 2006]. After washing and sonication to remove any debris and particulate matter [Gotliv et al., 2006], the sections were examined by either TOF-SIMS or scanning electron microscopy (SEM). The planes of the observed surfaces for sectioning and polishing were carefully selected to be as perpendicular as possible to the direction of the dentinal tubules, or at an angle of about $30^{\circ}$ to the tubule axis to expose the inside of the tubule surface. Some of the initial coronal sections were also taken whole, frozen at $-80^{\circ}$, crushed to a powder and then exposed to $5 \% \mathrm{NaOCl}$ at room temperature for 2 weeks to digest the ITD collagenous matrix and expose the mineralized PTD. The treated crude PTD was then fractionated on a stepwise aqueous sodium polytungstate density gradient [Weiner et al., 1999]. The particles collected at each step boundary were subjected to SEM, using a Hitachi S-3500 SEM in the high-vacuum mode, and SIMS analyses. For SEM, the samples were mounted on SEM pins using adhesive carbon tape and coated with a 6-nm layer of gold.

\section{Biochemical Analysis}

The PTD powder and various density fractions were demineralized in $0.8 \mathrm{M} \mathrm{HCl}$ at $4{ }^{\circ} \mathrm{C}$ and the soluble portion dialyzed against 


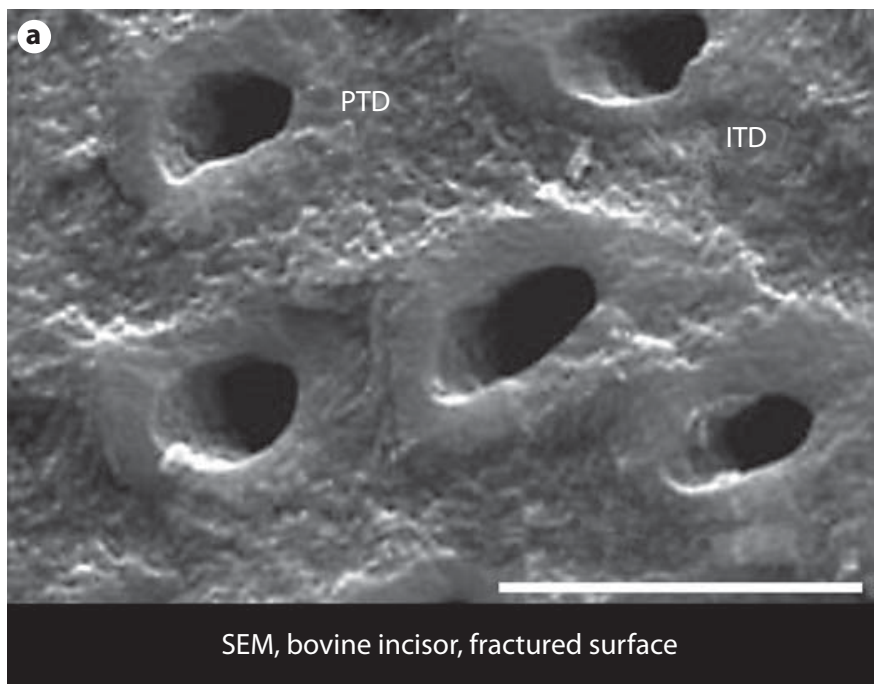

Fig. 1. SEM of fractured surface of coronal bovine incisor. a Standard SEM. Tubules are mainly cut perpendicular to the tubule direction. Note the relatively smooth and dense appearance of the PTD, but that there seems to be continuity of mineral at the PTDITD interfaces. Scale bar $=5 \mu \mathrm{m}$. b Standard SEM. Tubules are nearly parallel to the viewed surface so that their contents are evident. Note the relatively smooth and dense appearance of the PTD, but the inner surface is penetrated with many fenestrations. Scale bar $=5 \mu \mathrm{m}$. c Backscattering-mode SEM. Tubules are mainly cut perpendicular to the tubule direction. Note the enhanced density of the PTD indicating the higher mineral density compared to that in the ITD. Nevertheless, there still seems to be continuity of mineral crystals at the PTD-ITD interfaces. Scale bar = $10 \mu \mathrm{m}$.

deionized water using a 3500 MWCO Spectra/Por membrane. The retained material in the fractions shown to contain PTD by the SEM analyses were then subjected to a variety of procedures: SDS-PAGE; agarose/polyacrylamide gel electrophoresis; amino acid analysis; phosphate, sulfate sialic acid, neutral and amino sugar, uronic acid analyses; lectin binding; lipid extraction and analysis; all described in detail in Gotliv and Veis [2007].

\section{Results}

\section{SEM, Fractured Surfaces}

In order to obtain surfaces with the least artifactual modification, sections were collected after crudely fracturing the $-80^{\circ} \mathrm{C}$ coronal pieces, selecting those that had the best surface orientations. Figure la shows a section fractured from the crown of a bovine incisor tooth and
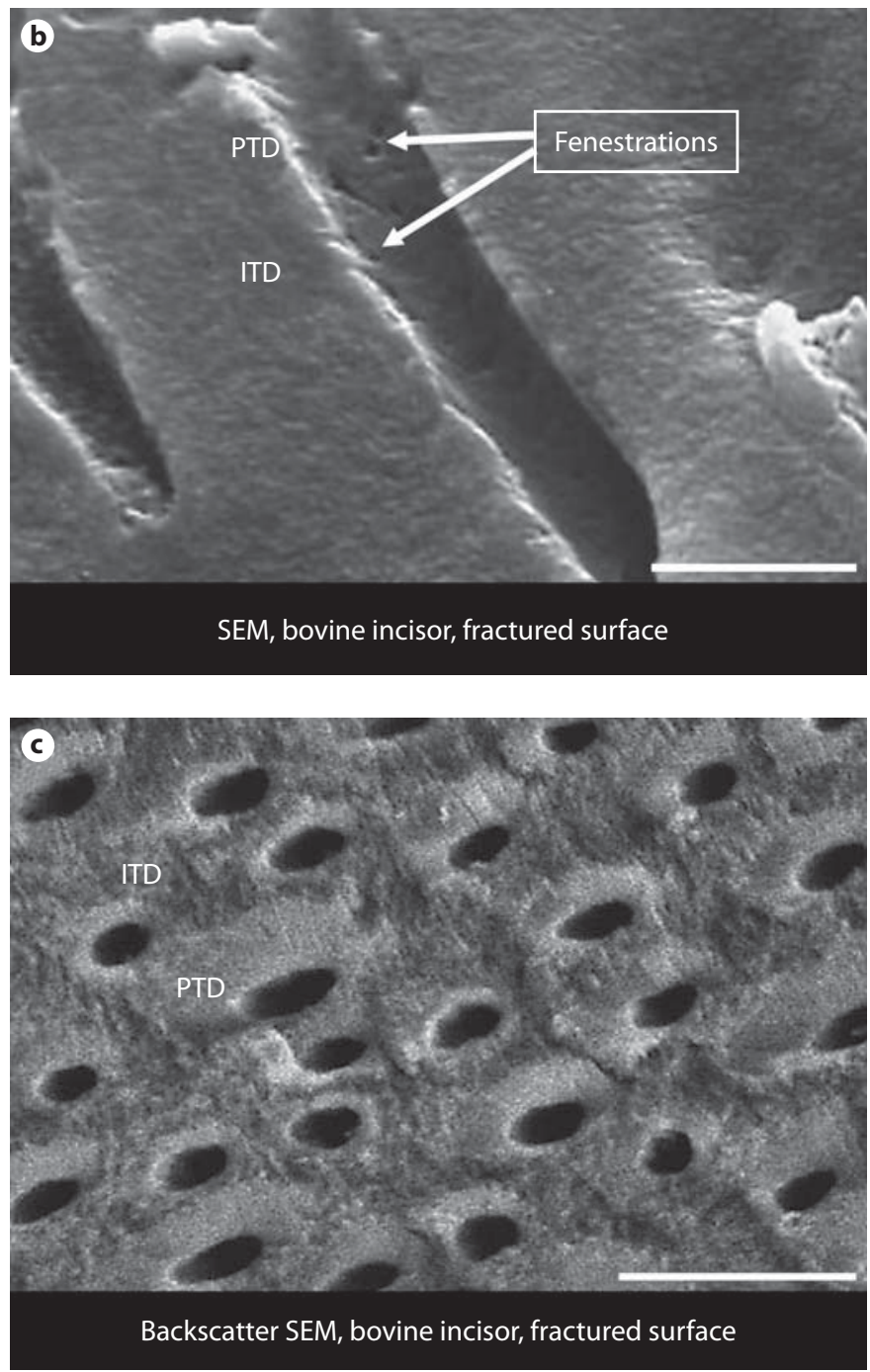

oriented so that the tubules are virtually perpendicular to the plane of view (also the tooth surface). The PTD is distinguished by its dense and smooth appearance compared to the more disorganized, granular appearance of the ITD. Figure 1b shows the same kind of fractured surface, but selected to be more parallel to the tubule long axis. Both the outer dense, smooth surface of the PTD is evident, and the inner tubule surface is exposed, showing the many fenestrations of the PTD. The uneven surface of the fractured tooth is evident, so it was important to establish that the smooth PTD collar was not an artifact of the coating for SEM. Therefore, the SEM section shown in figure la was reexamined in backscattering mode where the contrast between atoms of different atomic number can be seen. The PTD is seen to contain a higher concentration of atoms with higher atomic number, on average, 


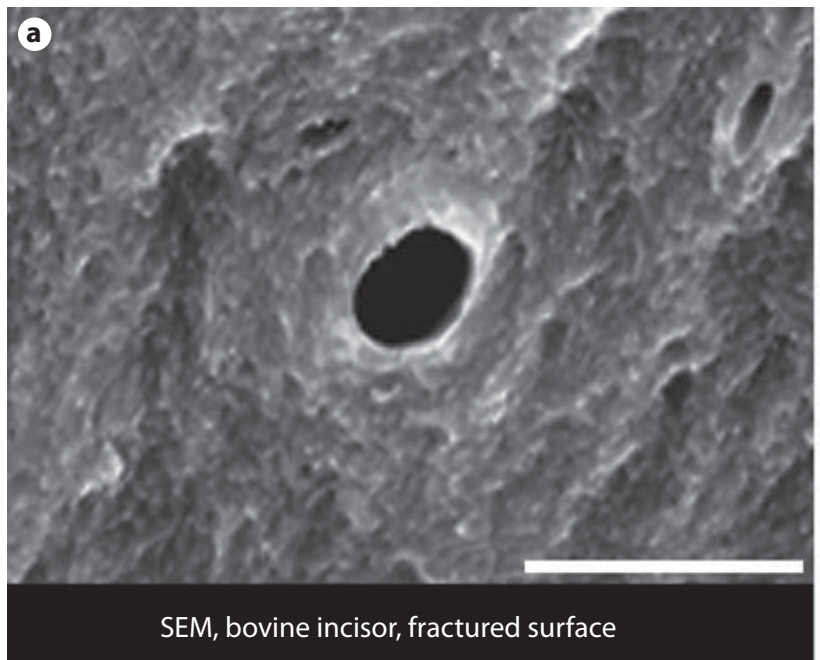

Fig. 2. SEM of fractured surface of bovine tooth after treatment with ethylenediamine to remove the majority of the accessible organic components. a Oriented perpendicular to the direction of the tubule. Edge effects still highlight the tubule, but the platy

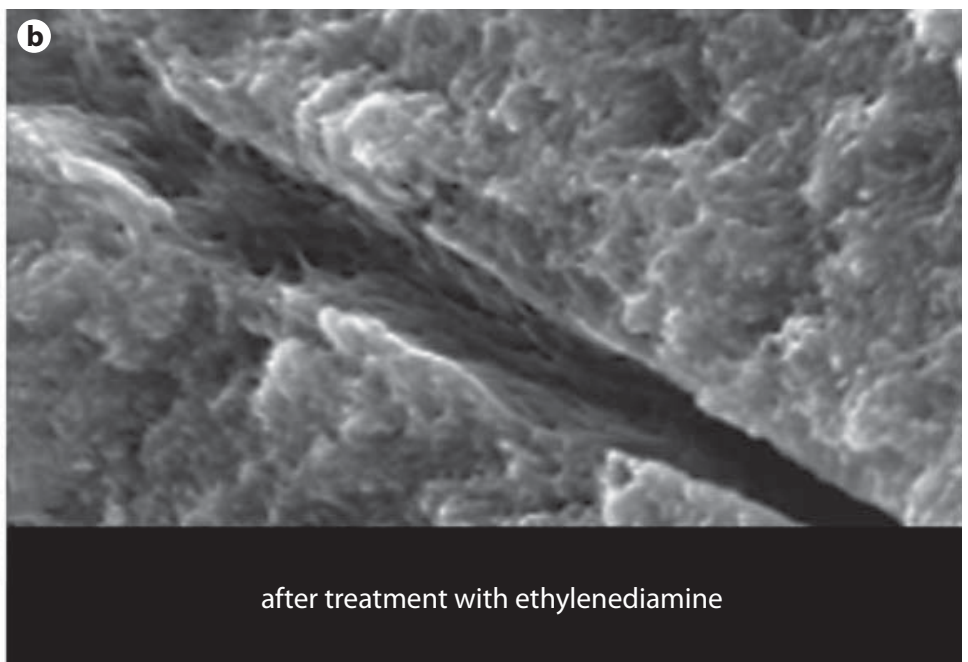

crystals of the PTD and their continuity with those in the ITD are evident. b Oriented along the tubule direction so as to reveal the inner tubule surface. The crystals are evident; the smooth material has been removed. Scale bar $=5 \mu \mathrm{m}$.
Fig. 3. SEM, polished dentin surface. Note that the very prominent PTD has been smoothed out and is not directly visible, but several other features are apparent and important. Looking at the tubule cross-sections, it is evident that few of the tubules are perpendicular to the surface and that, as shown by their elliptical shape, they are tilted in different directions and at different angles to the surface plane. The asterisks show odontoblastic processes, the broad arrows point to examples of retained PTD. The inset captures, at a $3 \times$ magnification from the main figure, a tubule in which the interior surface of the PTD was retained during the polishing. Note the many fenestrations of the PTD, distinct from the surrounding ITD.

than in the ITD (fig. 1c), a clear reflection of the higher Ca ion content and degree of mineralization of the PTD.

The organic constituents not protected by the mineral phase were removed by digestion with ethylenediamine [Skinner et al., 1972] and then viewed in tubule orientations as close as possible to those shown in figure 1a and b. In this case, the smooth-appearing material was re-

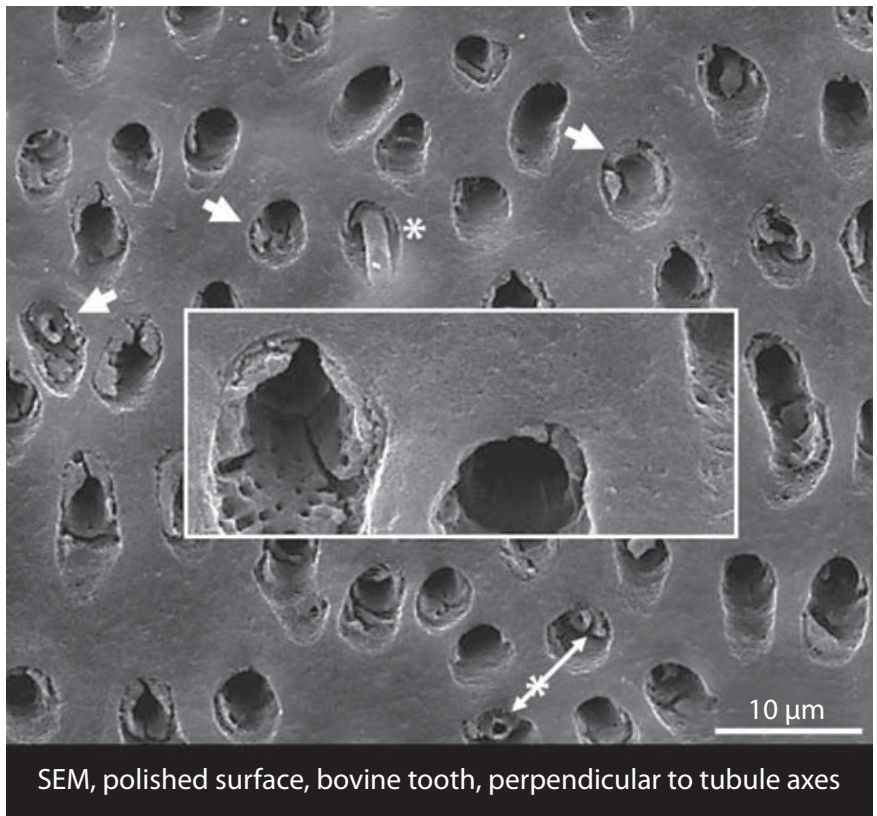

moved and the crystal plate orientations were revealed (fig. 2a and b). The platy character of the crystals was evident, but it was not possible to distinguish between the organization of crystals in the PTD and ITD. This was previously also shown to be the case in human dentin [Weiner et al., 1999]. There appears to be continuity between the PTD and ITD crystal orientations. 

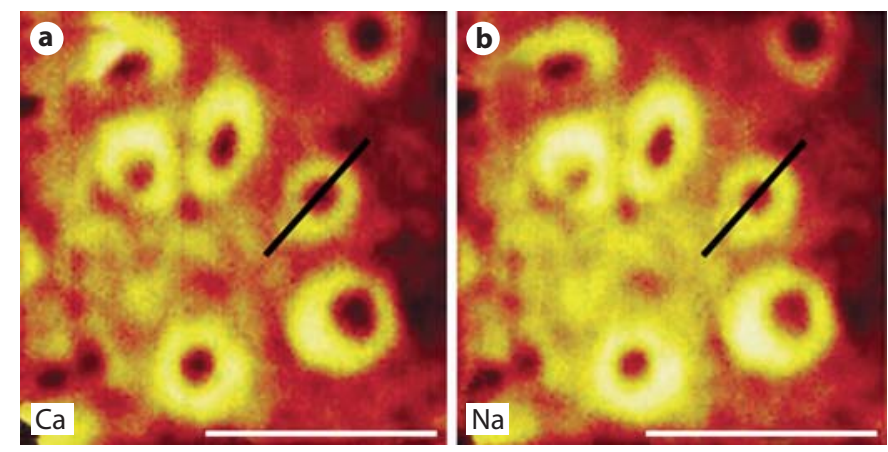

Fig. 4. TOF-SIMS of sodium and calcium ion distributions along a dentin surface perpendicular to tubule direction. The same surface is analyzed simultaneously for $\mathrm{Ca}-(\mathbf{a})$ and $\mathrm{Na}$-specific masses (b), plotted over a $30 \times 30 \mu \mathrm{m}$ area. c Plots of the relative intensity of the mass peak (Ca, $40.08 \mu \mathrm{mol}$; $\mathrm{Na}, 23 \mu \mathrm{mol})$ along the 7.5- $\mu \mathrm{m}$ line shown on the right in $\mathbf{a}$ and $\mathbf{b}$ for the same tubule and

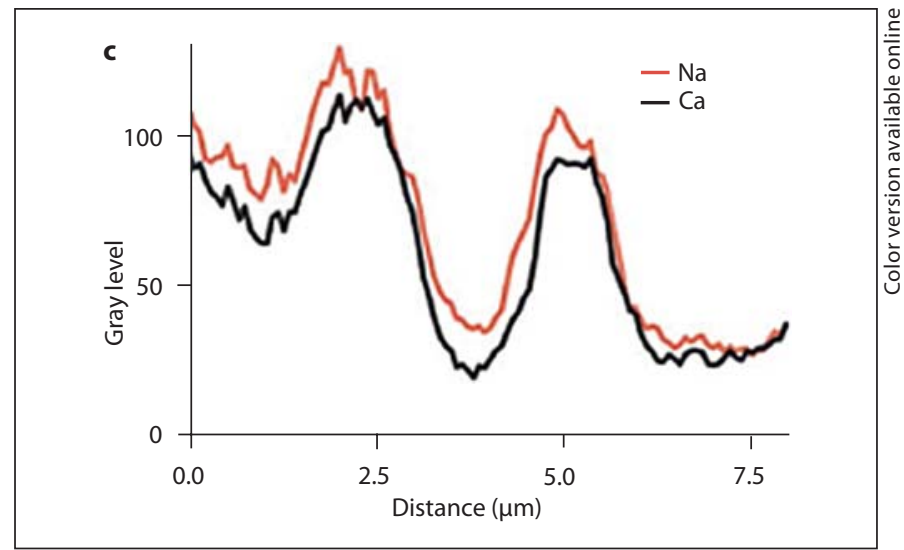

its surroundings. The tubule selected for measurement was the one with the most near-uniform annulus width. Since the tubules are not all perpendicular to the surface, the base lines on either side of the selected tubule reflect the uneven packing of the tubules within the section. Scale bars $=10 \mu \mathrm{m}$.
Fig. 5. TOF-SIMS distributions of amino acids in the PTD surface depicted in figure 4. The ion masses are shown in the lower corner of each panel, clearly separated from each other. The Ser + Ala peak is a reasonable measure of total protein content. Scale bars $=10 \mu \mathrm{m}$.
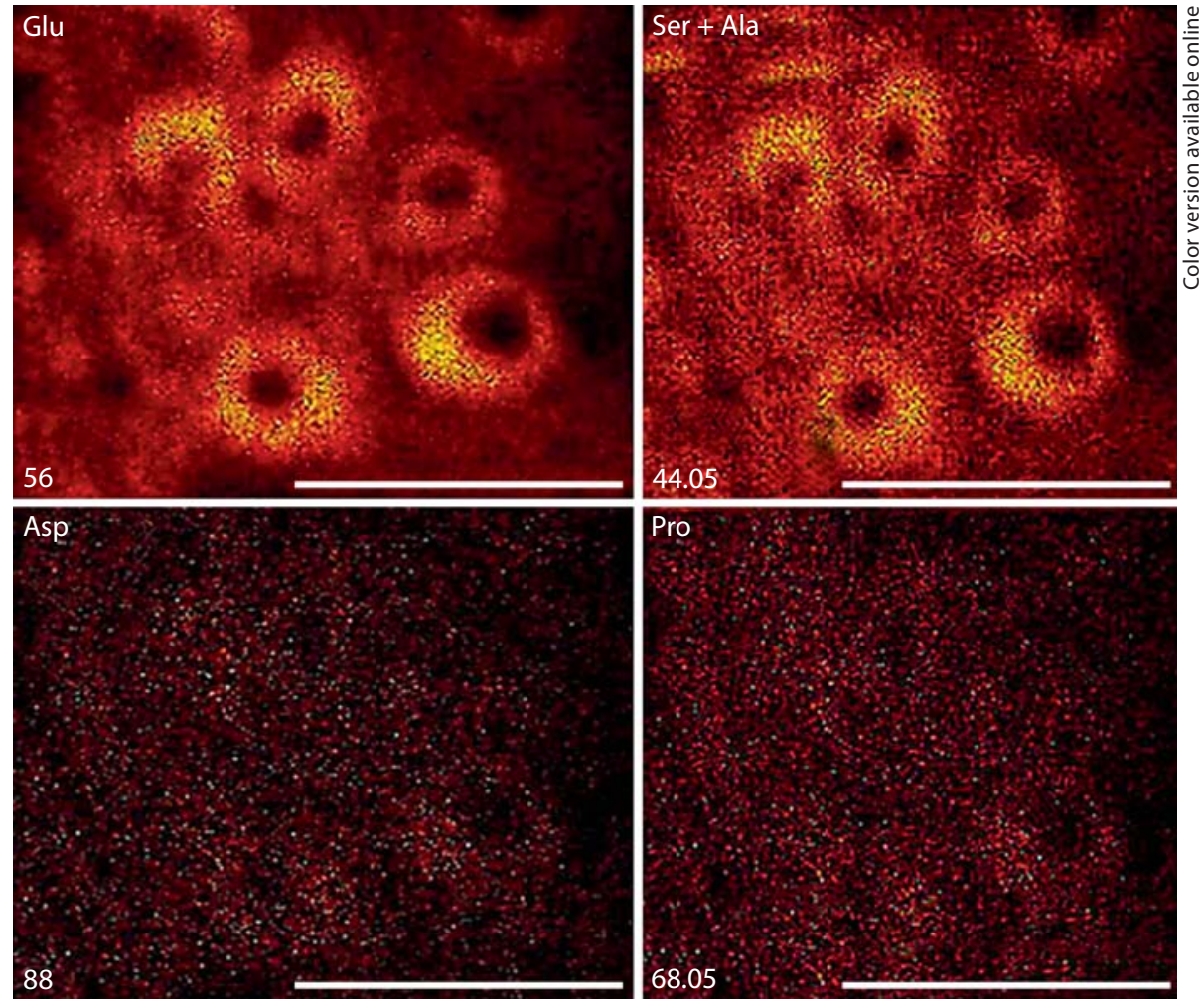

\section{SEM, Polished Surfaces}

TOF-SIMS surface composition mapping analysis requires that the scanned surface be quite flat. Hence, the exposed tooth surfaces to be studied must be polished.
This can be accomplished by the use of very fine grit polishes [Weiner et al., 1999; Gotliv et al., 2006; Gotliv and Veis, 2007], but one loses the architecture seen in the fractured surfaces. As shown in figure 3, some of the odon- 

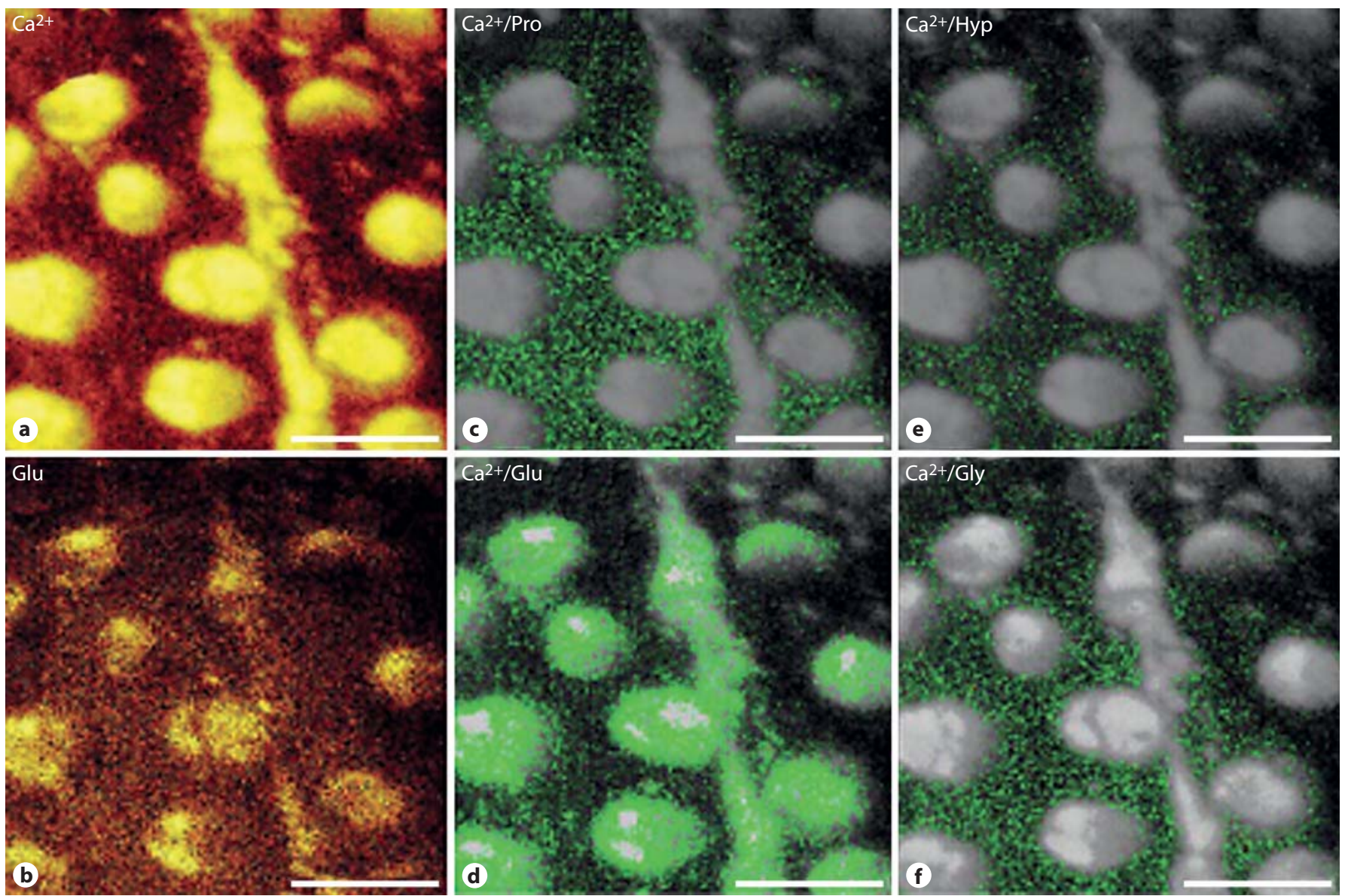

Fig. 6. Fractured tooth surface, no polish, selected area where plane of fracture was about 30 degrees from tubule direction. This exposed tubule surfaces, not tubule centers. All panels represent the same area, at the same magnification, and all data are from the same set of TOF-SIMS scans. a, b SIMS scans for Ca and Glu. c-f Image J colocalization of the indicated ions, denoted in green against gray background. Glu is prominently colocalized with $\mathrm{Ca}$ in the PTD surface, whereas the prominent mass ions arising from collagen, Pro, Hyp and Gly, are virtually restricted to colocalization in the ITD. toblastic processes are evident, and many of the tubules appear to retain a collar of material filling the space between the process and the ITD matrix. Some of the fragments inside the tubules may be artifacts from the polishing. However, when the polished surface is placed on the SIMS stage and sputtered to smooth the surface, a reasonable representation of the intact surface may be mapped.

\section{TOF-SIMS, Polished Surface Mapping}

The simple mineral ions are much more efficiently spalled from the surface, hence these peaks have higher number densities of fragments. The SIMS distributions of sodium $(\mathrm{Na}, 23 \mathrm{Da})$ and calcium ions $(\mathrm{Ca}, 40.08 \mathrm{Da})$ are shown in figure 4 for a section of polished surface corresponding to that shown in figure 3 . The brighter the color (see online version), the higher the number of ions of the selected mass at that position. The colors in figure $4 \mathrm{a}$ and $\mathrm{b}$ are relative to the highest intensity of the selected peak and are internally consistent for a given mass ion. The variations along the intensity traces in figure $4 \mathrm{c}$ show significant differences in the distribution of these ions across the entire surface, but do not provide quantitative or absolute values of the concentration of the particular ion. Not surprisingly, the region surrounding each tubule has a higher $\mathrm{Ca}$ content in the PTD than the ITD region, while the interiors of the tubules depicted have a mineralfree zone.

Of more interest is the distribution of the amino acid fragment ions. Figure 5 shows the distribution of Glu, Asp, Ser + Ala and Pro on the same surface area as depicted in figure 4 . As the masses become larger, the number of ion fragments spalled at a specified mass decreases, 
SEM
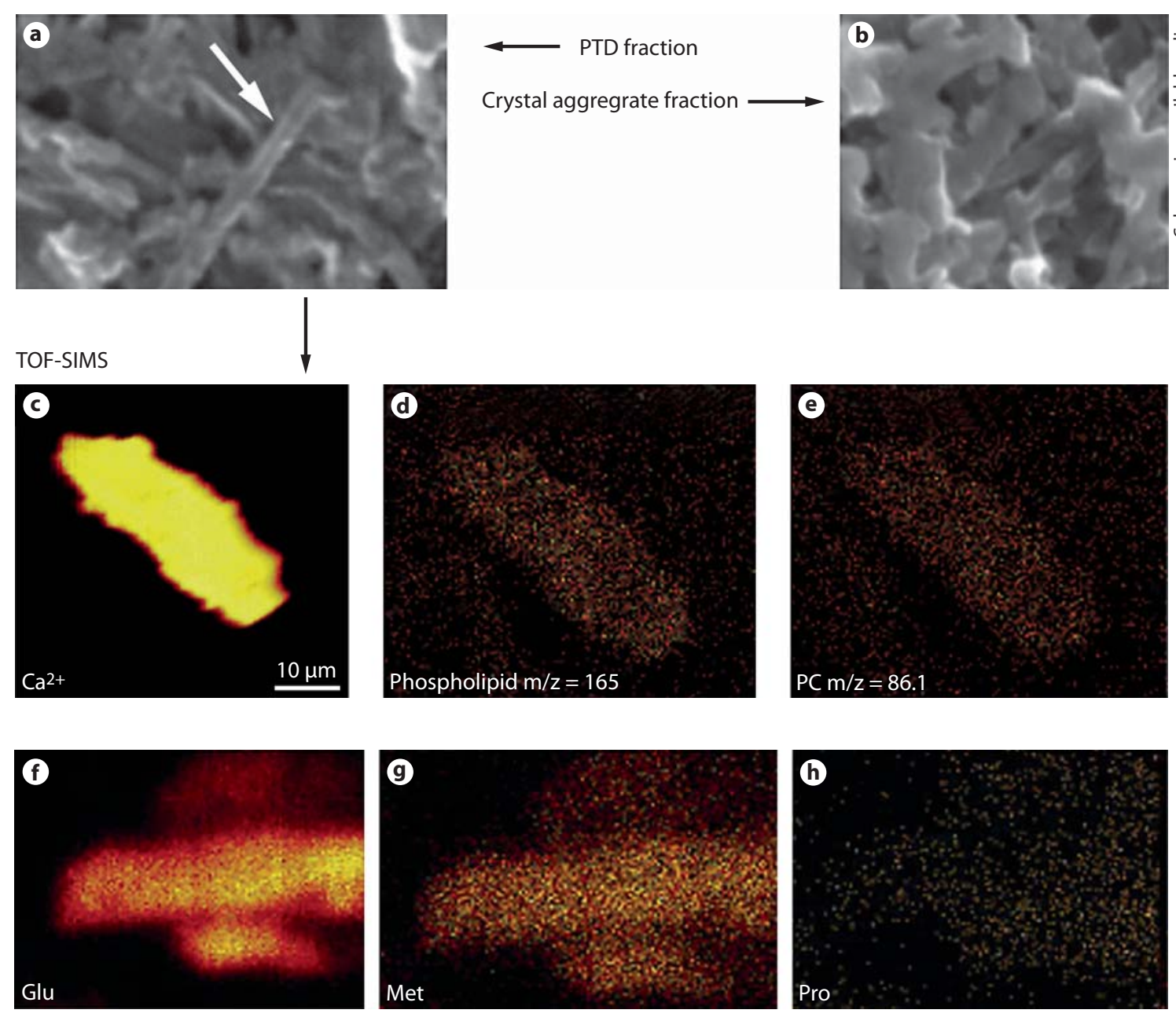

Fig. 7. SEM (a, b) and TOF-SIMS (c-h) of isolated intact PTD particles. These data demonstrate that the mineralized PTD surface shows the same composition as in cross-sections. It is virtually free of collagen, and has both phospholipids and acidic, glutamic acid-rich protein. All scans are at approximately the same scale as in c.

and the number of integrated counts of mass diminishes, thus it becomes more difficult to see these larger fragments. Nevertheless, it is clear that Glu fragment ions are produced in abundance at the PTD surface, while Asp fragment ions are not. NIH Image J colocalization procedures were used to examine the tubule surface with respect to $\mathrm{Ca}$ as well as Glu, Pro, Hyp and Gly. Figure 6 demonstrates that Glu is more abundant in the Ca-rich PTD surface than in ITD, where, in contrast, Pro, Gly and Hyp are more abundant than in PTD.

Although the PTD components can be seen inside the tubule, as in the inset of figure 3 , retention is rare. To circumvent this problem, the PTD fragments, obtained after digesting the ITD collagen and carrying out the density gradient fractionation, were simply deposited on a smooth silicon surface and subjected to SEM and TOF-SIMS. Although the surfaces were not smooth, the exposed surfaces were readily visualized. Figure 7 presents a composite of these data. SEM of the PTD fraction D showed it to be rod like (fig. 7a), whereas the more dense crystals of HAP had the typical thin platy appearance (fig. 7b).

The SIMS spectra showed the PTD particles to give a high Ca signal (fig. 7c), a fragment of 165 mass units (m/z) typical of most phospholipids (fig. 7d) and one particular to phosphatidylcholine (fig. 7e). Similar PTD particles, viewed in a separate experiment, showed the expected high Glu (fig. 7f), a reasonable signal for Met (fig. 7g), and the expected low Pro (fig. 7h). 


\section{Discussion}

The bovine PTD is clearly a distinct mineralized complex, separate from the mineralized collagen matrix of the ITD, and equally separate from the odontoblastic process and its cell membrane. Our earlier studies showed the PTD to contain a highly phosphorylated noncollagenous protein different from phosphophoryn [Weiner et al., 1999]; it is now evident that this protein owes its unusual properties of low solubility to its complexation with a mixture of phospholipids, forming a phospholipidphosphoprotein aggregate of high molecular mass that is not disaggregated during gel electrophoresis under denaturing conditions in SDS [Weiner et al., 1999]. Our examination of regular and backscatter SEM of specimens with virtually intact surfaces leads us to propose that the proteolipid-phospholipid complex provides a dense matrix that is nevertheless highly fenestrated with pores that may enable flux of fluids and ions between the ITD and odontoblastic processes. The negatively charged pore surfaces may serve as a selective regulator of ion transport between tubule and the ITD, a tissue that, although highly mineralized, is alive and in contact with the metabolic status of the host, just as is bone. At the same time, the proteolipid complex may participate in the PTD mineralization, just as has been suggested for the matrix vesicle lipids [Bonucci, 1967; Irving and Wuthier, 1968; Skinner et al., 1972; Peress et al., 1974; Wuthier, 1975]. In this regard it is notable that there appears to be a continuity of the mineral crystals in both the ITD and PTD (fig. 2). The outer PTD-ITD interface is not as sharply defined as the PTD-odontoblastic process membrane interface. However, when the PTD mineral is inadvertently removed during the polishing of the dried tooth surface, the underlying network of collagen fibers wound around the tubule in the ITD interface is sharp and clean (fig. 3). The abundant pores and cracks in the PTD suggest that the PTD does not play a mechanical structural supporting role in the dentin, but rather that its important role is, as suggested above, regulating transport of tubule constituents into the dentin, serving to keep it alive as a tissue [Boyan-Salyers and Boskey, 1980; Zabelinskii et al., 1984].

The second important conclusion is that the TOFSIMS approach may be the best way to examine the structure of the intact tooth, with three-dimensional reconstruction of the mineral content, coupled with chemical composition analysis. The key to the success of such study will be the resolution that can be achieved in static SIMS with the use of ever more narrowly focused incident beams, coupled with the intermittent use of slicing the surface with more intense surface milling beams to micropolish and expose defined surface depths.

\section{Acknowledgement}

Supported by grant DE-001374-45 from the NIDCR.

\section{References}

Bonucci, E (1967) Fine structure of early cartilage calcification. J Ultrastruct Res 20: 3350.

Boyan-Salyers, B.D., A.L. Boskey (1980) Relationship between proteolipids and calciumphospholipid-phosphate complexes in Bacterionema matruchotii calcification. Calcif Tissue Int 30: 167-174.

Dambach, S., M. Fartmann, C. Kriegeskotte, C. Brüning, S. Hellweg, H. Wiesmann, D. Lipinsky, H.F. Arlinghaus (2004) ToF-SIMS and laser-SNMS analysis of apatite formation in extracellular protein matrix of osteoblasts in vitro. Surf Interface Anal 36: 711-715.

Gotliv, B.A., A. Veis (2007) Peritubular dentin, a vertebrate apatitic mineralized tissue without collagen: role of a phospholipid-proteolipid complex. Calcif Tissue Int 81: 191-205.
Gotliv, B.A., J.S. Robach, A. Veis (2006) The composition and structure of bovine peritubular dentin: mapping by time of flight secondary ion mass spectroscopy. J Struct Biol 156: $320-333$.

Irving, J.T., R.E. Wuthier (1968) Histochemistry and biochemistry of calcification with special reference to the role of lipids. Clin Orthop Relat Res 56: 237-260.

Mantus, D.S., B.D. Ratner, B.A. Carlson, J.F. Moulder (1993) Static secondary ion mass spectrometry of adsorbed proteins. Anal Chem 65: 1431-1438.

Ostrowski, S.G., C. Szakal, J. Kozole, T.P. Roddy, J. Xu, A.G. Ewing, N. Winograd (2005) Secondary ion MS imaging of lipids in picoliter vials with a buckminsterfullerene ion source. Anal Chem 77: 6190-6196.

-Peress, N.S., H.C. Anderson, S.W. Sajdera (1974) The lipids of matrix vesicles from bovine fetal epiphyseal cartilage. Calcif Tissue Res 14: 275-282.
Samuel, N.T., M.S. Wagner, K.D. Dornfeld, D.G. Castner (2001) Analysis of poly(amino acids) by static time-of-flight secondary ion mass spectrometry (TOF-SIMS). Surf Sci Spectra 8: 163-184.

Skinner, H.C., E.S. Kempner, C.Y. Pak (1972) Preparation of the mineral phase of bone using ethylenediamine extraction. Calcif Tissue Res 10: 257-268.

Weiner, S., A. Veis, E. Beniash, T. Arad, J.W. Dillon, B. Sabsay, F. Siddiqui (1999) Peritubular dentin formation: crystal organization and the macromolecular constituents in human teeth. J Struct Biol 126: 27-41.

Wuthier, R.E. (1975) Lipid composition of isolated epiphyseal cartilage cells, membranes and matrix vesicles. Biochim Biophys Acta 409: 128-143.

Zabelinskii, S.A., L.F. Pomazanskaia, E.V. Chirkovskaia (1984) Brain proteolipids in representatives of different vertebrate classes. Zh Evol Biokhim Fiziol 20: 239-245. 